\title{
Melatonin attenuates postovulatory oocyte dysfunction by regulating SIRT1 expression
}

\author{
Qingling Yang ${ }^{1,2, *}$, Shanjun Dai ${ }^{1,2, *}$, Xiaoyan $\operatorname{Luo}^{1,2, *}$, Jing Zhu ${ }^{1,2}$, Fangyuan $\mathrm{Li}^{1,2}$, Jinhao Liu ${ }^{1,2}$, \\ Guidong Yao ${ }^{1,2}$ and Yingpu Sun ${ }^{1,2}$ \\ ${ }^{1}$ Reproductive Medical Center, First Affiliated Hospital of Zhengzhou University, Zhengzhou, China and \\ ${ }^{2}$ Henan Province Key Laboratory for Reproduction and Genetics, Zhengzhou, China \\ Correspondence should be addressed to Y Sun; Email: syp2008@vip.sina.com \\ *(Q Yang, S Dai and X Luo contributed equally to this work)
}

\begin{abstract}
The quality of postovulatory metaphase II oocytes undergoes a time-dependent deterioration as a result of the aging process. Melatonin is considered to be an anti-aging agent. However, the underlying mechanisms of how melatonin improves the quality of postovulatory aged oocytes remain largely unclear. In this study, by using mouse model, we found that there were elevated reactive oxygen species levels and impaired mitochondrial function demonstrated by reduced mitochondrial membrane potential and increased mitochondrial aggregation in oocytes aged $24 \mathrm{~h}$, accompanied by an increased number of meiotic errors, unregulated autophagy-related proteins and early apoptosis, which led to decreased oocyte quality and disrupted developmental competence. However, all of these events can be largely prevented by supplementing the oocyte culture medium with $10^{-3} \mathrm{M}$ melatonin. Additionally, we found that the expression of sirtuin family members (SIRT1, 2 and 3) was dramatically reduced in aged oocytes. In addition, in vitro supplementation with melatonin significantly upregulated the expression of SIRT1 and antioxidant enzyme MnSOD, but this action was not observed for SIRT2 and SIRT3. Furthermore, the protective effect of melatonin on the delay of oocyte aging vanished when the SIRT1 inhibitor EX527 was used to simultaneously treat the oocytes with melatonin. Consistent with this finding, we found that the postovulatory oocyte aging process was markedly attenuated when the oocytes were treated with the SIRT1 activator SRT1720. In conclusion, our data strongly indicate that melatonin delays postovulatory mouse oocyte aging via a SIRT1-MnSOD-dependent pathway, which may provide a molecular mechanism support for the further application of melatonin in the assisted reproductive technology field.

Reproduction (2018) 156 81-92
\end{abstract}

\section{Introduction}

The prophase I oocyte resumes meiosis during maturation when it is released from the ovary, then the oocyte is later arrested again at the metaphase II (MII) after germinal vesicle breakdown and awaits fertilization by spermatozoa (Sun et al. 2009). There is an approximately $10-\mathrm{h}$ period, called the optimal window, in which fertilization can occur. If no fertilization occurs during this time frame, MII oocytes will progressively undergo a process of quality deterioration, referred to as postovulatory aging (Tarin et al. 1999, Lord \& Aitken 2013), which results in compromised oocyte function, as displayed by abnormal zona hardening, mitochondrial dysfunction, elevated reactive oxygen species (ROS) levels, spindle dysfunction and loss of chromosomal integrity (Ducibella et al. 1990, Xu et al. 1997, Takahashi et al. 2003, Wakayama et al. 2004, Lord \& Aitken 2013, Lord et al. 2013), which ultimately led to limited oocyte competence, abnormal embryo development, congenital malformations and reduced pregnancy outcomes after fertilization (Tarin et al. 1999, Kosubek et al. 2010). However, the detailed mechanisms of postovulatory oocyte aging are still largely unknown. Additionally, there are no effective strategies for delaying the oocyte aging process in vitro or in vivo during infertility treatment in clinical practice with assisted reproductive technologies (ARTs).

Melatonin ( $\mathrm{N}$-acetyl-5-methoxy tryptamine) is produced by the pineal gland and has been proved to play a key role in regulating reproductive activity in both animals (Reiter 1991) and humans (Reiter 1998). Previous studies suggest that melatonin can regulate the release of gonadotropin, which then regulates reproductive activities in animals, but the regulatory mechanism of melatonin on the hypothalamic-pituitary-gonadal axis is unclear in humans (Reiter 1980, 1998, Roy \& Belsham 2002). In contrast, some studies found that the melatonin concentration in preovulatory follicular fluid in 
humans was significantly higher than that in serum in the same individuals (Brzezinski et al. 1987, Ronnberg et al. 1990). Further, the melatonin levels in follicular fluid are associated with both the quantity and quality of oocytes in in vitro fertilization (IVF) (Tong et al. 2017), suggesting that melatonin may play a key role in follicular development in humans. Importantly, an increasing number of studies has reported that melatonin can improve the oocyte quality and subsequent embryonic development in mammals as a free radical scavenger and antioxidant (Papis et al. 2007, Rodriguez-Osorio et al. 2007, Tamura et al. 2008, Kang et al. 2009, Shi et al. 2009, Lord et al. 2013, Wang et al. 2013, 2014, 2017b, Coelho et al. 2015). Additionally, some studies found that melatonin could modulate the gene transcription of antioxidant enzymes (Tomas-Zapico \& Coto-Montes 2005, Han et al. 2017, Wang et al. 2017 b).

Although the intracellular signals that control postovulatory oocyte aging have not been well defined, previous studies have shown that the postovulatory aging of mammalian oocytes is inherently linked to oxidative stress and that the ROS content increases in a time-dependent manner as a result of the imbalance between production and consumption during postovulatory oocyte aging (Galano et al. 2011), indicating that oxidative stress plays an integral role in curtailing the structural and functional integrity of oocytes. Recent studies have shown that melatonin supplementation prevents postovulatory oocyte aging and improves embryonic development in mammals (Lord et al. 2013, Dai et al. 2017, Wang et al. 2017b). However, the underlying mechanisms regarding how melatonin improves the quality of postovulatory aged oocytes remain unclear. Therefore, the aim of this study was to attempt to investigate the effect of melatonin on the delay in postovulatory oocyte aging and the mechanisms involved.

In the present study, the results showed that in vitro supplementation of melatonin in the culture medium significantly delayed postovulatory oocyte aging by reducing the ROS levels, mitochondrial dysfunction, meiotic errors, autophagy and apoptosis levels. Additionally, for the first time, we found that the protective effect of melatonin on postovulatory oocytes was mediated by the SIRT1-MnSODdependent pathway.

\section{Materials and methods}

Six-week-old ICR female mice were purchased from Charles River Laboratories. All the mice had free access to food and water and were kept under controlled conditions with a constant temperature range, from 20 to $25^{\circ} \mathrm{C}$ with a 12 -h light/darkness cycle. All experiments were approved by the institutional review board and received approval from the First Affiliated Hospital of Zhengzhou University.

\section{Oocyte collection and treatment}

To collect the MII oocytes, mice were stimulated by an intraperitoneal injection of $7.5 \mathrm{IU}$ of pregnant mares serum gonadotropin, and 7.5 IU human chorionic gonadotropin (hCG) was injected to induce superovulation. The mice were killed $12-14 \mathrm{~h}$ after the hCG was used. The cumulus-oocyte complexes (COCs) were obtained by breaking the oviductal ampullae. The cumulus cells were removed by pipetting in the IVF (Vitrolife Sweden AB, V.Frölunda, Sweden) medium including 5\% human serum albumin (Irvine Scientific, Santa Ana, CA, USA) with $0.1 \%$ hyaluronidase (Sigma), and we then set the time to $0 \mathrm{~h}$ when the COCs were collected. In vitro aged MII oocytes were used in experiments at 0, 6, 12 and $24 \mathrm{~h}$. For the melatonin treatment, the treated oocytes were incubated in the IVF medium with a final concentration of $10^{-7}, 10^{-5}$ or $10^{-3} \mathrm{M}$ melatonin (Sigma) respectively.

\section{IVF and embryo culture}

Male mice aged 15-20 weeks were killed and their spermatozoa were released from the dissected epididymis. Then, the spermatozoa were transferred to IVF medium supplemented with BSA (Sigma) for $1 \mathrm{~h}\left(37^{\circ} \mathrm{C}, 5 \% \mathrm{CO}_{2}\right)$ to achieve capacitation. Then, spermatozoa were used to fertilize the ovulated oocytes at a concentration of $1 \times 10^{5} / \mathrm{mL}$ in $60 \mu \mathrm{L}$ of IVF medium at $37^{\circ} \mathrm{C}, 5 \% \mathrm{CO}_{2}$. Four-cell and blastocyst embryo rate were calculated on day 3 and day 6 respectively.

\section{ROS detection}

To determine the ROS content in oocytes, the procedure was conducted according to the handbook for the ROS Detection Kit (Beyotime Institute of Biotechnology, Hangzhou, China) with some modification, as follows: the MII oocytes were incubated in a culture solution of G-MOPS(Vitrolife) with a final concentration of $10 \mu \mathrm{M}$ DCFH (dichlorofluorescein) diacetate for $20 \mathrm{~min}$ at $37^{\circ} \mathrm{C}$ in the dark. Then, the oocytes were washed three times in the IVF medium and the oocytes were observed, captured and measured by using a confocal microscope with the same scanning settings (Zeiss LSM 700).

\section{Immunofluorescence analysis}

All the oocytes were fixed in $4 \%$ paraformaldehyde for 30 min and permeabilized with $0.5 \%$ Triton $\mathrm{X}-100$ in PBS for $20 \mathrm{~min}$, followed by blocking for $1 \mathrm{~h}$ with $1 \%$ BSA in PBS at room temperature. The oocytes were then incubated with anti- $\alpha$-tubulin monoclonal antibody (Sigma) (1:200), antiSIRT1 (Proteintech, Wuhan, China) $(1: 100)$ or anti-MnSOD (Proteintech) $(1: 100)$ overnight at $4{ }^{\circ} \mathrm{C}$, and then the oocytes were incubated with Alexa Fluor 488 goat anti-mouse or Alexa Fluor 555 donkey anti-rabbit secondary antibody for $2 \mathrm{~h}$ at room temperature. After washing three times in PBS,

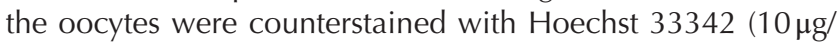
$\mathrm{mL}$ ) for $10 \mathrm{~min}$ and placed on glass slides. For mitochondrial staining, oocytes were incubated in IVF medium supplemented with $250 \mathrm{nM}$ MitoTracker Red (Invitrogen) for $30 \mathrm{~min}$ at $37^{\circ} \mathrm{C}$, followed by washing three times in PBS; then, the oocytes were 
mounted on glass slides and captured under a laser scanning microscope (Zeiss LSM 700).

\section{Annexin V staining}

After the cumulus cells were removed by pipetting in the IVF medium with $0.1 \%$ hyaluronidase, the oocytes were collected and washed three times in PBS; then, the oocytes were stained in binding buffer containing 10\% Annexin V-FITC (Beyotime, Hangzhou, China). Finally, the Annexin V-positive oocytes were mounted and captured by a laser scanning microscope (Zeiss LSM 700).

\section{Mitochondrial membrane potential measurement $(\Delta \Psi \mathrm{m})$}

The $\Delta \Psi \mathrm{m}$ was measured by using the mitochondrial inner membrane potential dye 5, 5', 6, 6'-tetrachloro-1, 1', 3, 3'-tet raethylbenzimidazolylcarbocyanine iodide (JC-1) (Beyotime), according to the manufacturer's instructions. Briefly, after washing in the PBS twice, oocytes were incubated in $100 \mu \mathrm{L}$ of working solution with a final concentration of $10 \mu \mathrm{M} \mathrm{JC}-1$ at $37^{\circ} \mathrm{C}$ in $5 \% \mathrm{CO}_{2}$ for $20 \mathrm{~min}$ in the dark. The oocytes then were washed in PBS three times and imaged immediately in green and blue fluorescence channels using a laser scanning microscope. The red and green fluorescence intensities were determined as the sum of fluorescence in the oocyte, and the mitochondrial membrane potential was calculated as the ratio of red-to-green fluorescent pixels.

\section{RNA isolation and real-time PCR}

Total RNA was isolated from 50 fresh or aged oocytes by using a micropurification kit (Qiagen), according to manufacturer's instructions. First-strand cDNA was synthesized using cDNA synthesis kit (TaKaRa, Kusatsu, Japan). Real-time RT-PCR was performed on 96-well plates using Quantstudio 12K Flex (Applied Biosystems) using SYBR Green reaction mix (Qiagen). The primers sequences of Sirt1, Sirt2, Sirt3, Mnsod and Gapdh are shown in Table 1. Relative gene expression was calculated by the $2^{-\Delta \Delta \mathrm{Ct}}$ method.

\section{Western blot analysis}

The oocytes were lysed with the lysis buffer from the protein extraction kit (Sangon Biotech, Shanghai, China) and quantified by using a protein quantitation kit (Bio-Rad), according to the manufacturer's instructions. The denatured protein was separated by $10 \%$ SDS-PAGE gel and transferred to PVDF membrane. After blocking with $5 \%$ defatted dry milk diluted by TBST (Tris-buffered saline with Tween20) for $1 \mathrm{~h}$, the membranes were incubated with primary antibodies of LC3 (CST, CA, USA) (1:1000), P62 (Abcam) (1:1000) and SIRT1 (Proteintech) (1:500) overnight at $4{ }^{\circ} \mathrm{C}$ respectively. On the next day, the membranes were incubated with HRP (horseradish peroxidase)-conjugated goat anti-mouse or goat anti-rabbit secondary antibodies for $1 \mathrm{~h}$ at room temperature after washing six times in TBS. The protein bands were viewed by using the enhanced chemiluminescence detection system (Bio-Rad).

\section{Statistical analysis}

For each experiment, at least three replicates were performed unless specified otherwise. Data are shown as the mean \pm S.E.M. and analyzed by one-way ANOVA, followed by least significant difference (LSD) post hoc test and the chi-squared test were used as appropriate, all the data were analyzed using Statistical Program for Social Sciences (SPSS Inc., version 17.0). $P<0.05$ was considered significant.

\section{Results \\ In vitro administration of melatonin prevents morphological changes in postovulatory aged MII oocytes}

Amorphological analysiswascarriedouttodetectwhether melatonin could delay postovulatory oocyte aging. The MII oocytes were aged in vitro, and the morphological changes were recorded at 0 (control), 6, 12 and $24 \mathrm{~h}$, and the results indicated that the oocyte abnormality rate was increased in a time-dependent manner and was shown by degeneration and fragmentation (Fig. 1A and $\mathrm{B}$ ). There were approximately $60 \%$ of oocytes that showed a morphological defect after aging for $24 \mathrm{~h}$, which was significantly higher than that of control at $\mathrm{Oh}$ (Fig. 1B), so this time point was chosen for subsequent experiments. To investigate the effects of melatonin on the protective action of delayed oocyte aging, the oocytes were cultured for $24 \mathrm{~h}$ in the IVF medium with different concentrations of melatonin $\left(10^{-7}, 10^{-5}\right.$ and $\left.10^{-3} \mathrm{M}\right)$. We found that the oocyte abnormality rate was significantly lower when cultured in the IVF medium with $10^{-3} \mathrm{M}$ melatonin compared to the aged oocytes incubated with $10^{-7}$ and $10^{-5} \mathrm{M}$ melatonin (Fig. 1C), which indicated that melatonin prevents postovulatory aging in mouse oocytes in a dose-dependent manner. Therefore, the $10^{-3} \mathrm{M}$ melatonin concentration was chosen for the following experiments.

Table 1 Primer sequences.

\begin{tabular}{lll}
\hline & \multicolumn{1}{c}{ Forward } & \\
\hline Sirt1 & 5'-TATCTATGCTCGCCTTGCGG-3' & 5'-CGGGATATATTTCCTTTGCAAACTT-3' \\
Sirt2 & 5' -TCTGCCACTACTTCATCCGC-3' & 5'-ATGTGTAGAAGGTGCCGTGG-3' \\
Sirt3 & 5'-TATGGGCTGATGTGATGGCG-3' & 5'-AGTCGGGGCACTGATTTCTG-3 \\
Mnsod & 5'-ATTAACGCGCAGATCATGCA-3' & 5'-TGTCCCCCACCATTGAACTT-3' \\
Gapdh & 5'-TGGCAAAGTGGAGATTGTTGCC-3' & 5'-AAGATGGTGATGGGCTTCCCG-3' \\
\hline
\end{tabular}


A
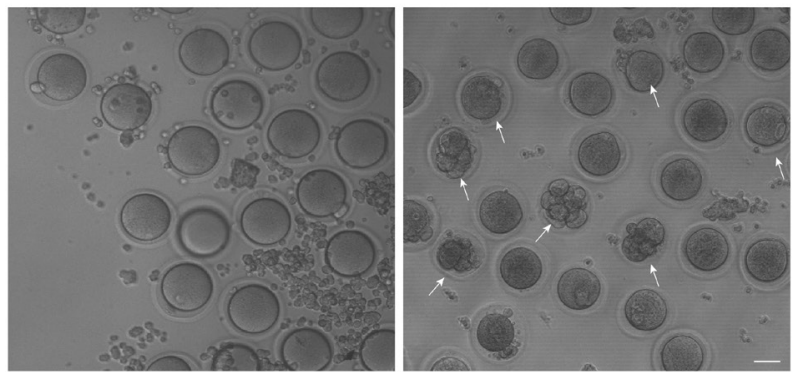

B

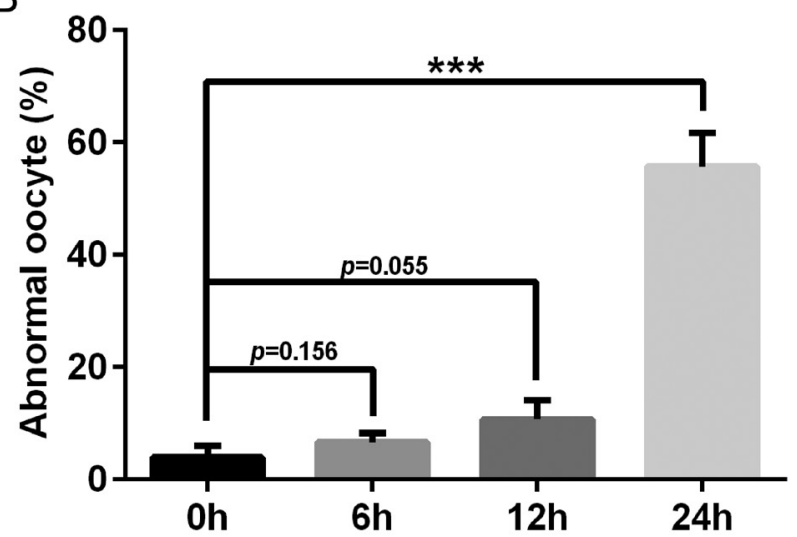

C

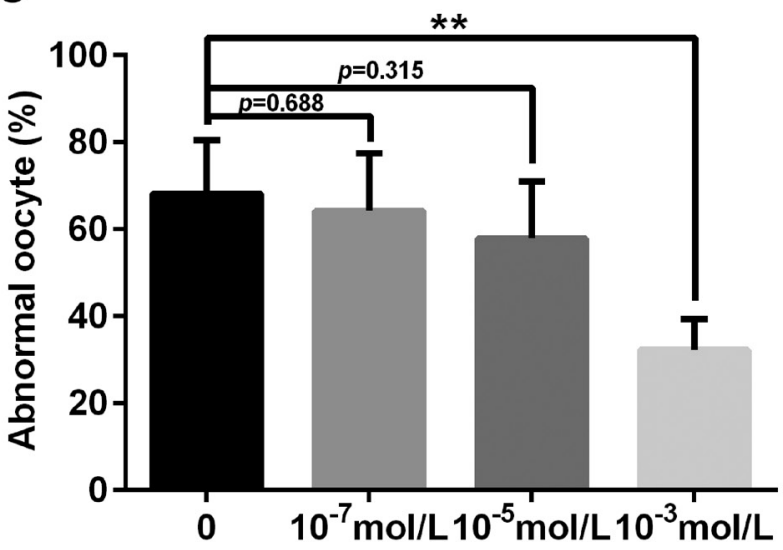

Figure 1 Effects of melatonin on the morphological change of postovulatory aged oocytes. (A) Representative images of fresh (left) and aged abnormal oocytes (right, white arrow). (B) Abnormal oocyte rates of oocytes aged for $0,6,12$ and $24 \mathrm{~h}$ respectively $(n=100$ for each group). (C) Abnormal oocyte rates of aged oocytes treated by different melatonin concentrations ( $n=100$ for each group). $* * P<0.01 ; * * * P<0.001$. Scale bar: $50 \mu \mathrm{m}$.

\section{Melatonin suppresses ROS production and spindle dysfunction in postovulatory aged MII oocytes}

Oxidative stress has been considered a key mechanism underlying cellular aging, so we next investigated whether the ROS content was decreased in aged oocytes treated with melatonin. The results showed that compared to controls, the ROS level was significantly higher in aged oocytes; however, when the oocytes were exposed to $10^{-3} \mathrm{M}$ melatonin, the ROS level in oocytes was markedly decreased compared with that of aged oocytes (Fig. 2A and B). Considering that an elevated ROS level is usually associated with meiotic defects in oocytes, denuded oocytes were also examined for spindle morphology. As shown in Fig. 2C, the results revealed that fresh MII oocytes displayed a typical barrel-shaped spindle with well-aligned chromosomes on the equatorial plate (with only $9.6 \%$ abnormality Fig. 2D). While in aged oocytes, $41.6 \%$ of oocytes had defective spindles, shown as disorganized, which was significantly higher than the percentage of fresh oocytes. As expected, the proportion of abnormal spindles was significantly lower in oocytes treated by melatonin (16.7\%) than that in aged oocytes (Fig. 2D). Taken together, these results indicate that the in vitro administration of melatonin indeed markedly reduces meiotic defects in postovulatory aged oocytes.

\section{Melatonin reduces the mitochondrial dysfunction in postovulatory aged MII oocytes}

Mitochondria are also an important subcellular target during the oocyte aging process. In the next step, $\Delta \Psi \mathrm{m}$ was determined in aged oocytes by staining with the inner membrane potential dye JC-1. In addition, the mitochondrial membrane potential was represented by the ratio of red-to-green fluorescence in the oocyte. The results showed that the mean ratio of red-to-green fluorescence of aged oocytes was significantly lower than that of fresh oocytes, indicating that the aged oocytes have lower mitochondrial membrane potential (Fig. 3A and $\mathrm{B}$ ); whereas with melatonin supplementation, the mitochondrial membrane potential was significantly increased compared to 24-h aged oocytes (Fig. 3A and B). The mitochondrial distributions during MII oocyte aging were also examined in this study. As shown in Fig. 3C, the mitochondria displayed an evenly distributed pattern throughout the cytoplasm in fresh MII oocytes. In contrast, the mitochondria were aggregated in clusters in the aged MII oocytes. Importantly, this ratio of oocytes with an abnormal mitochondrial distribution pattern was significantly reduced in oocytes treated with melatonin (Fig. 3D). These results suggested that melatonin may play an important role in reducing the mitochondrial dysfunction during postovulatory oocyte aging in vitro.

\section{Melatonin prevents autophagy and apoptosis during postovulatory oocyte aging}

It is known that aging induces oxidative stress, which accelerates oocyte apoptosis; therefore, the early apoptosis events were detected by the staining of Annexin V. As shown in Fig. 4A, the green fluorescent circle located on the external cellular membrane of the oocyte was clearly observed in aged oocytes, but only 
A

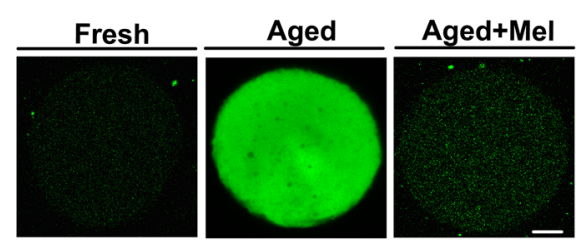

C

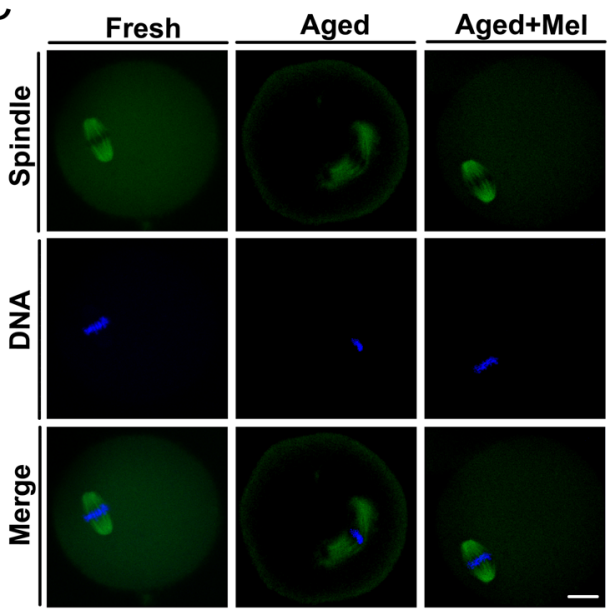

$\mathrm{B}$

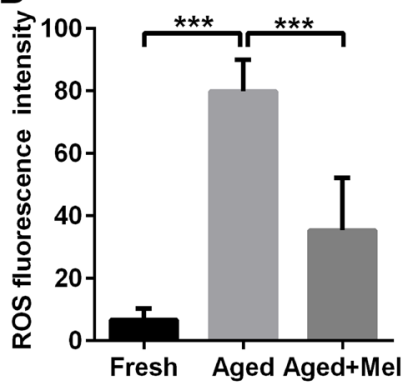

D

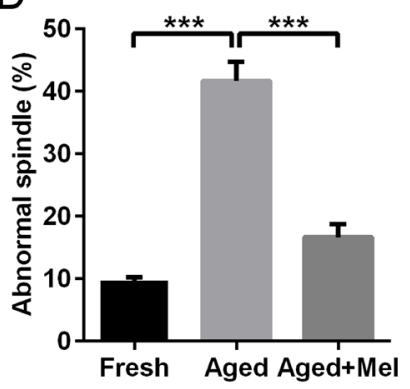

Figure 2 Effects of melatonin on ROS generation and spindle morphology in postovulatory aged oocytes. (A) Representative images of ROS fluorescence (green) in oocytes from fresh group (Fresh), aged group (Aged) and aged oocytes treated with melatonin group (Aged+Mel). (B) Quantification of the relative ROS levels in oocytes of the three different groups ( $n=35$ for Fresh, $n=36$ for Aged and $n=33$ for Aged+Mel). (C) Spindle morphology in fresh, aged and melatonintreated oocytes. (D) The proportions of abnormal spindles in fresh $(n=108)$, aged $(n=106)$ and melatonin-treated oocytes $(n=112)$. Data are expressed as the mean \pm S.E.M. from three independent experiments. ${ }^{* * *} P<0.001$; Scale bar: $20 \mu \mathrm{m}$. ROS, reactive oxygen species. a weak signal was detected in fresh oocytes. Analysis of the proportion of the Annexin V-positive oocytes showed that only approximately $17.3 \%$ of oocytes displayed early apoptosis in the fresh oocytes, whereas $76.3 \%$ were positively stained in the aged oocytes. However, the proportion decreased to $45.6 \%$ in the melatonintreated group (Fig. 4B).
On the other hand, elevated ROS could also lead to increased autophagy, to further explore whether the autophagy level was altered in the process of postovulatory oocyte aging, the induction of autophagy was also measured in oocytes through western blot, which showed that aged oocytes exhibited a higher autophagy level, demonstrated by higher expression of
A
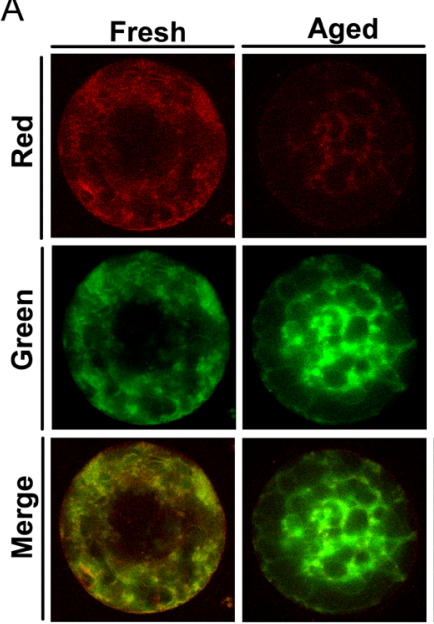

C
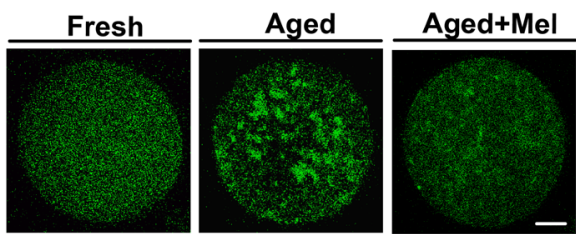

www.reproduction-online.org
B

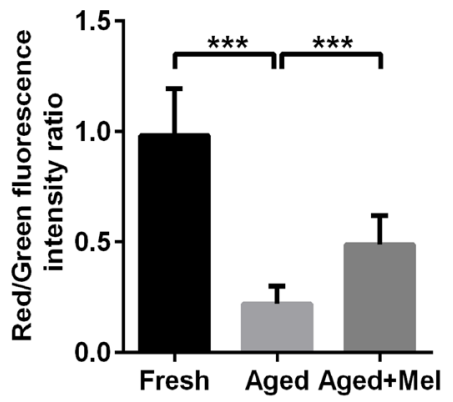

D

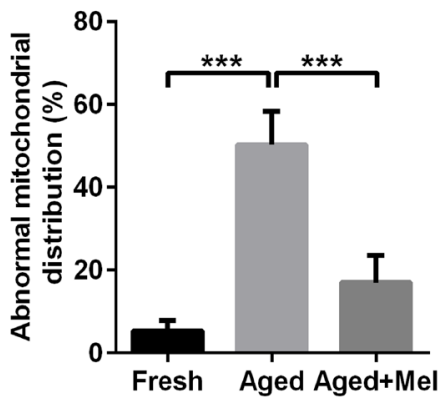

Figure 3 Effects of melatonin on $\Delta \Psi \mathrm{m}$ and mitochondria distribution in postovulatory aged oocytes. (A) Representative examples of oocytes from fresh, aged and melatonintreated aged groups. Oocytes were stained with JC-1, where red fluorescence indicates high $\Delta \Psi \mathrm{m}$ and green indicates low $\Delta \Psi \mathrm{m}$. (B) Ratio of red to green fluorescence, an indicator of mitochondrial membrane potential ( $n=28$ for Fresh, $n=31$ for Aged and $n=34$ for Aged+Mel) (C) Representative images of mitochondria distribution in oocytes from fresh, aged and aged+Mel groups. (D) The proportion of abnormal mitochondrial distribution in these three groups $(n=38$ for Fresh, $n=40$ for Aged and $n=36$ for Aged+Mel). Data are expressed as the mean \pm S.E.M. from three independent experiments. ${ }^{* * *} P<0.001$; Scale bar: $20 \mu \mathrm{m}$. $\Delta \Psi \mathrm{m}$, mitochondrial membrane potential. 
A
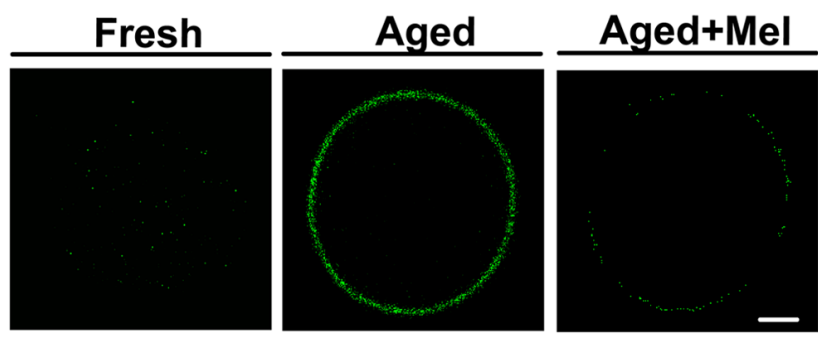

B
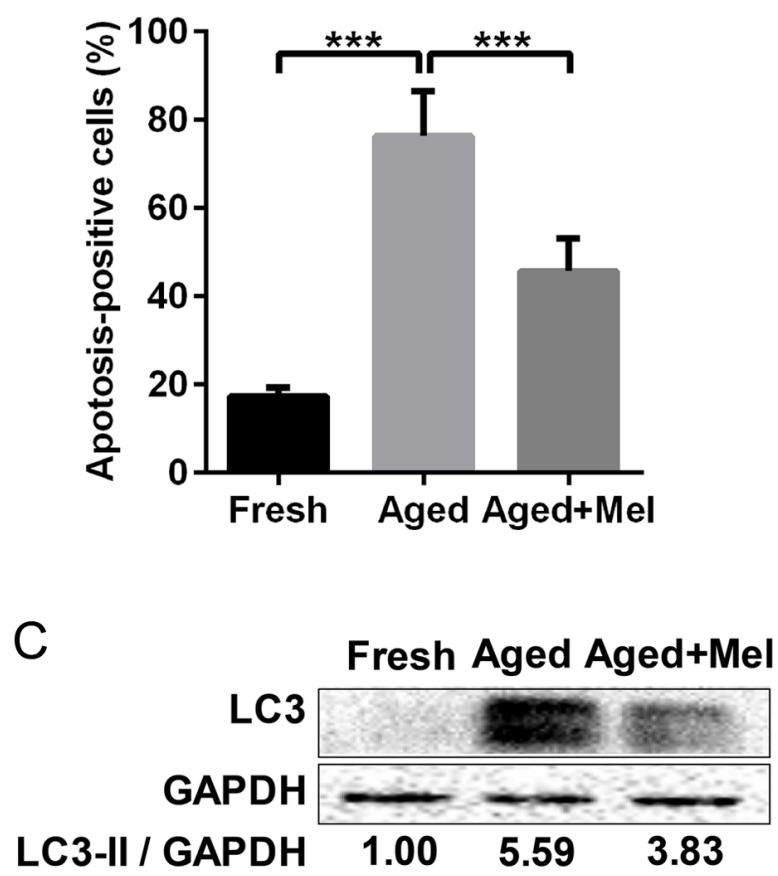

D

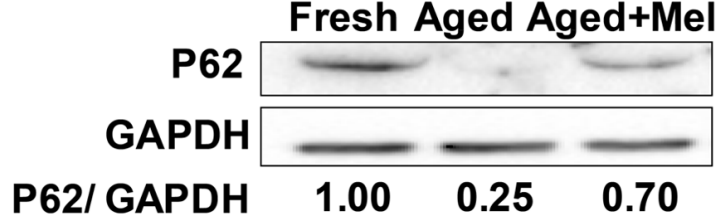

Figure 4 Effects of melatonin on early apoptosis and autophagy in postovulatory aged oocytes. (A) Representative images of apoptosis in control, aged and melatonin-treated aged oocytes. Oocytes were stained with Annexin V-FITC. (B) The ratio of early apoptosis was recorded in fresh, aged and melatonin-supplemented oocytes ( $n=45$ for Fresh, $n=48$ for Aged and $n=52$ for Aged+Mel). Data are expressed as the mean \pm S.E.M. from three independent experiments. Western blot was used to detect the autophagyrelated protein LC3 (C) and P62 (D), for each band, at least 300 oocytes were used. The band intensity was calculated using the Image J software, and the ratio of LC3-II or P62/GAPDH expression was normalized; values are indicated. $* * * P<0.001$; Scale bar: $20 \mu \mathrm{m}$.
LC3-II (microtubule-associated protein-1 light chain 3, which is recruited to both the outer and inner surfaces of the autophagosomal membrane and remains on the completed autophagosome until it fuses with a lysosome) (Fig. 4C) and lower expression of P62 (a substrate of autophagy, which is degraded by the autophagylysosomal pathway) (Fig. 4D) than that of fresh oocytes, but melatonin significantly alleviated the process.

\section{Melatonin improves the early embryonic developmental potential of postovulatory aged MII oocytes}

All the above results showed that melatonin could delay the aging of postovulatory oocytes in mouse. Therefore, we wanted to know if melatonin could improve the early embryonic developmental potential of aged oocytes. The IVF was carried out for fresh, aged and melatonintreated aged oocytes. The results showed that $82.3 \%$ (Fig. 5A and B) of the oocytes could develop into fourcell embryos and that approximately $61.3 \%$ (Fig. 5C and D) could develop to the blastocyst stage in fresh oocytes. For 24-h aged oocytes, the proportions of fourcell and blastocyst embryos were decreased to 30.3 and $9.3 \%$ respectively (Fig. 5B and D). However, in the melatonin-treated aged oocyte group, the proportions of four-cell (44.3\%) and blastocyst formation (28.6\%) were significantly increased (Fig. 5B and D). Taken together, these results suggest that melatonin can delay the aging and improve the early embryonic developmental potential of postovulatory oocytes.

\section{Melatonin elevates SIRT1 and MnSOD expression during postovulatory MII oocyte aging}

Melatonin's protective action during oocyte aging has been reported in previous studies (Lord et al. 2013, Wang et al. 2017b); however, the detailed mechanisms have still been unclear until now, which further prompted us to investigate the potential mechanisms involved. One published study showed that SIRT1, 2 and 3 are downregulated during oocyte aging in vivo or in vitro (Zhang et al. 2016). In addition, previous studies have shown that melatonin could regulate the sirtuin expression in different cells and mammal models (Han et al. 2016, 2017, Guo et al. 2017, Liu et al. 2017, Nopparat et al. 2017, Song et al. 2017, Tamura et al. 2017, Zhai et al. 2017). Therefore, we speculated that SIRT1, 2 and 3 might be the important downstream targets in the melatonin delaying postovulatory MII oocyte aging process. To validate this speculation, the oocytes were collected, and the mRNA expressions of Sirt1, Sirt2 and Sirt3 were analyzed. The results showed that compared to fresh oocytes, the mRNA level of Sirt1, 2 and 3 were all dramatically decreased in 24-h aged oocytes 
A
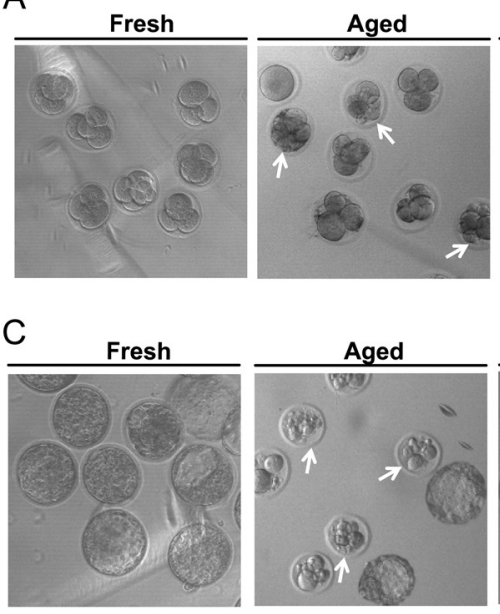

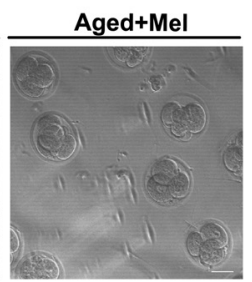

B

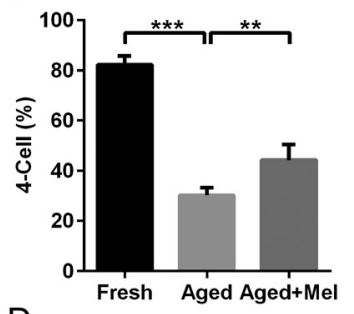

D

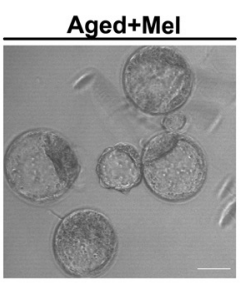

Figure 5 The development of embryos from fresh, aged and melatonin-treated oocytes. (A) Representative images of four-cell embryos from fresh, aged and melatonin-treated aged oocytes. White arrows show the abnormal developed embryo. (B) The percentage of embryos that successfully progressed to four-cell embryos in the three groups. (C) Representative images of blastocyst embryos from fresh, aged and melatonin-treated oocytes. White arrows show the abnormal developed embryo (D) The proportion of embryos that successfully progressed to blastocyst embryos $(n=105$ for Fresh, $n=97$ for Aged and $n=104$ for Aged+Mel). Data are expressed as the mean \pm S.E.M. from three independent experiments. ${ }^{* *} P<0.01$; ${ }^{* * *} P<0.001$; Scale bar: $50 \mu \mathrm{m}$.
(Fig. 6A, B and C). In addition, the expression levels of Sirt 2, 3 were only slightly regulated in oocytes treated by melatonin, with no significant difference (Fig. 6B and C). However, compared to aged oocytes, the Sirt 1 mRNA expression was markedly increased in melatonin-treated oocytes (Fig. 6A). This foundation was further verified by immunofluorescence (Fig. 6D and E) and western blot analysis of SIRT1 (Fig. 6F).

A previous study reported that SIRT1 could upregulate the antioxidant protein, MnSOD, to protect cells against the oxidative stress (Kops et al. 2002). Then, we wanted to know if MnSOD expression was altered accordingly by treatment with melatonin in aged oocytes. The expressions of Mnsod mRNA and protein were determined by real-time PCR, immunofluorescence and western blot. As expected, compared to the aged oocytes, the expression levels of MnSOD were significantly higher in the oocytes treated by melatonin (Fig. 6G, H, I and J). Collectively, these data indicate that melatonin may stimulate MnSOD expression to protect the oocyte against the oxidative stress and delay oocyte aging by regulating the SIRT1 expression.

\section{Melatonin delays postovulatory MII oocyte aging via a SIRT1-MnSOD-dependent pathway}

As shown earlier, melatonin has a protective effect during oocyte aging that is apparently mediated by a SIRT1MnSOD-dependent pathway. Hence, we hypothesized that melatonin attenuates postovulatory mouse oocyte aging via a SIRT1-MnSOD-dependent pathway. To validate this hypothesis, the SIRT1 inhibitor EX527 $(100 \mathrm{nM})$ and melatonin were added to the culture medium simultaneously. When the oocyte aged for $24 \mathrm{~h}$, the SIRT1 and MnSOD expression was determined by western blot. As shown in Fig. 7A, compared to the 24-h oocytes, the increased expression of SIRT1 and MnSOD was no longer observed. Furthermore, the ROS content and spindle morphology were evaluated in these groups. Compared to melatonin-only treatment during oocyte aging, the ROS level and abnormal spindle morphology rate were also significantly increased when oocytes were treated by melatonin and SIRT1 inhibitor simultaneously (Fig. 7B, C, D and E).

Additionally, we wanted to know whether artificially increasing the expression of SIRT1 would delay the postovulatory aging process in vitro. The SIRT1 activator SRT1720 was used in the followed experiments. When SRT1720 (100 nM) was added to the culture medium during oocyte aging, the ROS level and spindle morphology were analyzed. We found that compared with the aged oocytes, the SIRT1 activator could significantly upregulate the SIRT1 and MnSOD expression (Fig. 8A). Consequently, the ROS levels and meiotic defect rate were dramatically decreased (Fig. 8B, $\mathrm{C}, \mathrm{D}$ and $\mathrm{E})$. In summary, we conclude that melatonin could delay postovulatory MII oocyte aging through a SIRT1-MnSOD-dependent pathway.

\section{Discussion}

In recent years, the proportion of new babies produced by ART has been gradually increasing, especially in Western countries (Giritharan et al. 2007). Additionally, postovulatory oocyte aging is an important negative factor for oocyte quality during the ART treatment process, and it is well established that the oocyte quality determines the embryo's subsequent developmental potential after fertilization (Wang \& Sun 2007). Early studies reported that the postovulatory oocyte aging process is regulated by a series of molecular mechanisms (Miao et al. 2009, Tatone et al. 2015) and that increased oxidative stress is the basis of all events that occurred in the postovulatory oocyte (Takahashi et al. 2003, Lord \& Aitken 2013, Zhang et al. 2016). Therefore, antioxidative compounds 
A

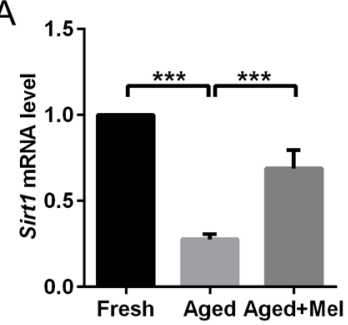

D

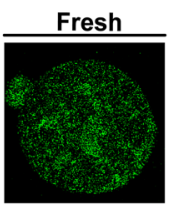

E

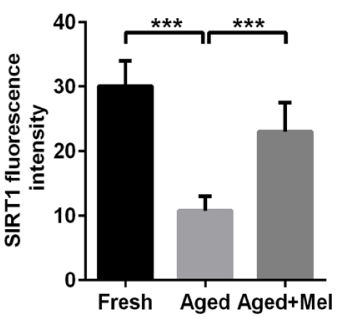

F

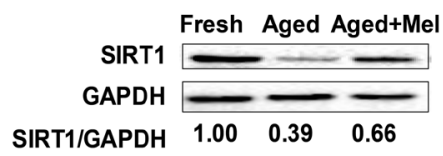

C
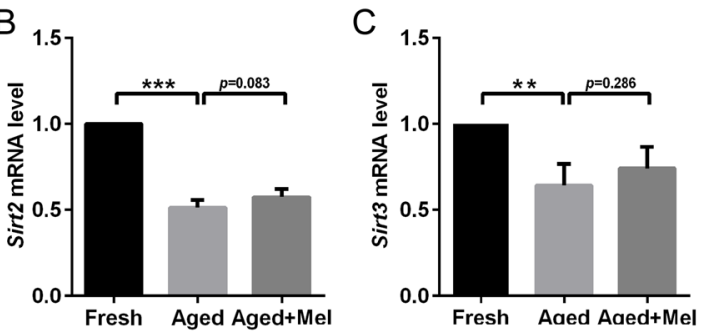

G

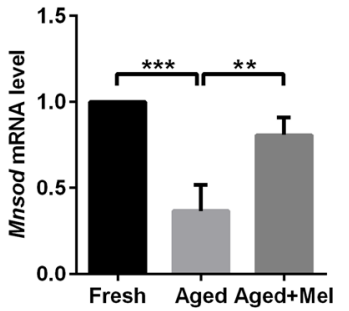

$\mathrm{H}$

I
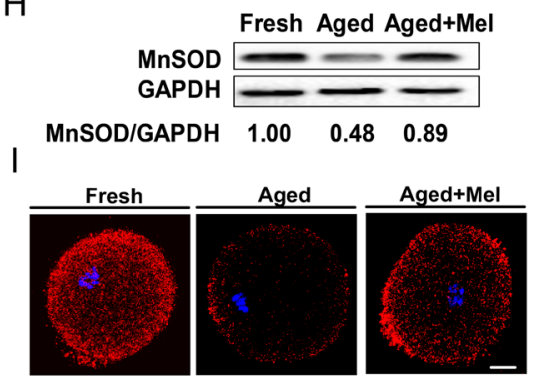

$\mathrm{J}$

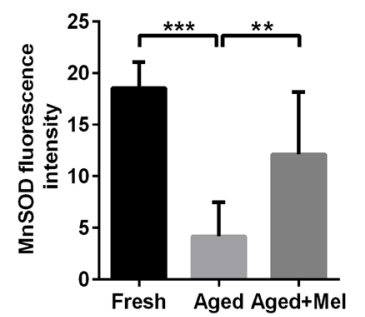

Figure 6 Effects of melatonin on the expressions of SIRT1 and MnSOD in postovulatory aged oocytes. Expression of Sirt1 (A), Sirt2 (B) and Sirt3 (C) mRNA in oocytes from fresh, aged and melatonin-treated aged oocytes groups. The mRNA expression of Sirt1, 2 and 3 was determined by real-time PCR analysis. $n=50$ for each group at one experiment with at least three independent experiments. (D) Representative immunofluorescence images of the SIRT1 in oocytes from fresh, aged and melatonin-treated aged oocytes groups. (E) The expression of SIRT1 was calculated as the total green fluorescence in each oocyte ( $n=35$ for Fresh, $n=34$ for Aged and $n=37$ for Aged+Mel). (F) Western blot analysis of the SIRT1 in fresh, aged and melatonin-treated oocytes. For each band, at least 300 oocytes were used. (G) The expression of Mnsod mRNA detected by real-time PCR in oocytes from the three different groups. $(\mathrm{H})$ Western blot analysis of the MnSOD in fresh, aged and melatonin-treated oocytes. For each band, at least 300 oocytes were used. (I) Representative immunofluorescence images of MnSOD in oocytes from the three groups. (J) MnSOD levels were calculated as the total red fluorescence in each oocyte ( $n=34$ for Fresh, $n=30$ for Aged and $n=32$ for Aged+Mel). For each band, at least 300 oocytes were used. Data are expressed as the mean \pm S.E.M. from three independent experiments for mRNA and immunofluorescence detections. ${ }^{* *} P<0.01 ;{ }^{* * *} P<0.001$. Scale bar: $20 \mu \mathrm{m}$.

have been studied to examine their protective effects during oocyte aging, including quercetin (Wang et al. 2017a), resveratrol (Liu et al. 2013) and melatonin (Lord et al. 2013, Dai et al. 2017, Wang et al. 2017b). Among these, melatonin has been shown to increase the IVF rate and early embryonic development in mice (Ishizuka et al. 2000) and humans (Tamura et al. 2008), indicating that melatonin may be relatively promising for application in clinical treatment. However, the underlying molecular mechanisms that account for these observations have remained largely unknown.
In this study, we used mouse oocytes as a model to determine the effects of melatonin on delaying postovulatory oocyte aging. The results demonstrated that melatonin could act as a powerful inhibitor to delay oocyte aging by reducing the ROS level, mitochondrial dysfunction, spindle morphology, autophagy and apoptosis (Figs 2, 3 and 4), and finally improve oocyte quality (Fig. 1) and early embryonic development (Fig. 5). Most importantly, for the first time, we found that the defects caused by postovulatory aging could be restored, at least partially, based on the regulation of SIRT1 expression by melatonin. 


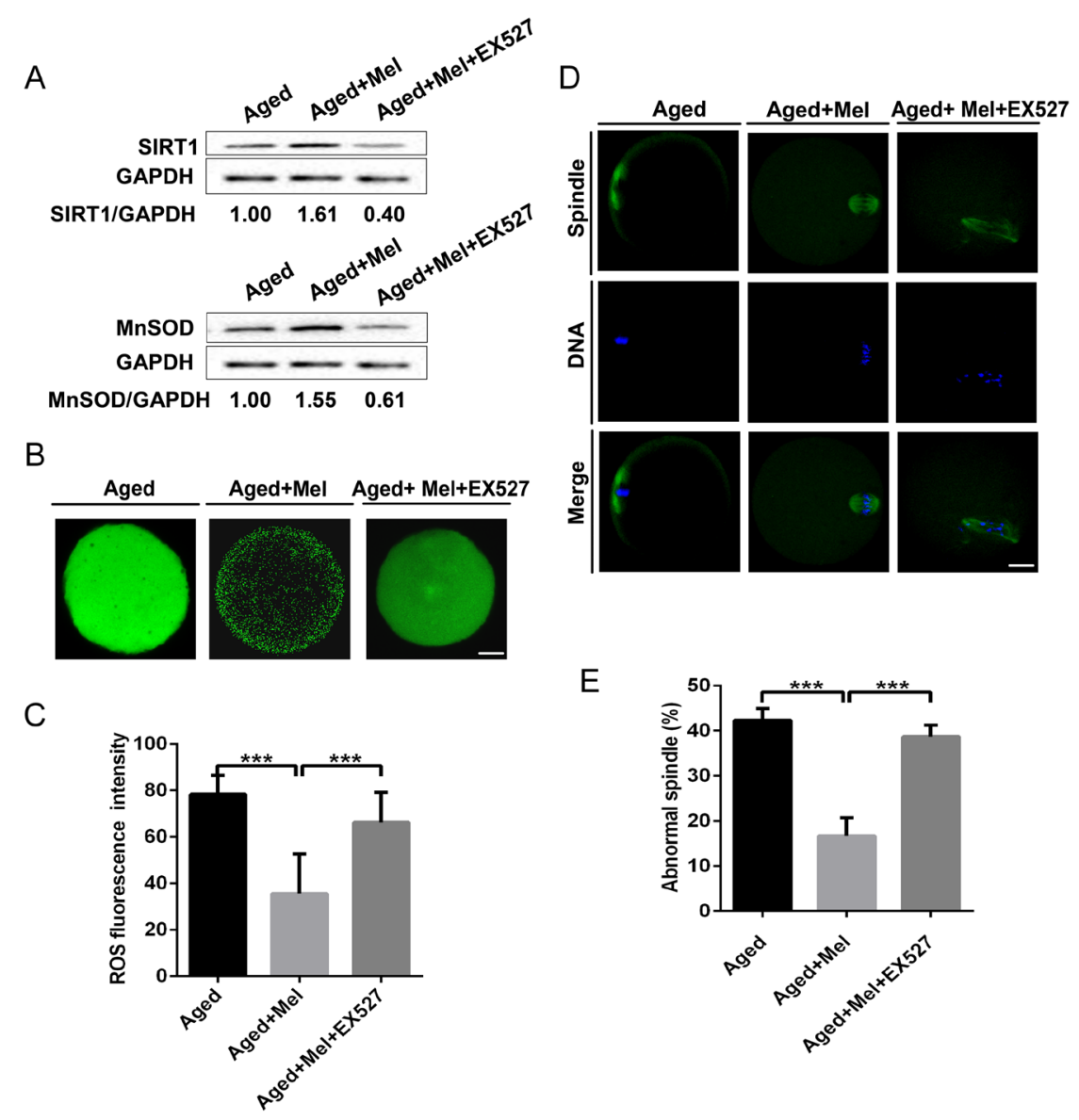

Figure 7 Inhibition of the SIRT1 abolishes the effects of melatonin on reducing ROS level and abnormal spindle morphology in postovulatory aged oocytes. (A) SIRT1 and MnSOD protein expressions in oocytes were analyzed by western blot from aged, melatonin-treated aged and melatonin+EX527-treated aged groups. For each band, at least 300 oocytes were used. (B) ROS representative immunofluorescence images of oocytes from the three different groups. (C) ROS fluorescence pixel intensity in oocytes ( $n=30$ for Aged, $n=27$ for Aged + Mel, and $n=25$ for Aged+Mel+EX527). (D) Representative images of spindle morphology in aged, melatonin-treated aged and melatonin + EX527-treated MII oocytes. (E) The proportion of abnormal spindles in these groups respectively ( $n=105$ for Aged, $n=97$ for Aged + Mel and $n=87$ for Aged+Mel+EX527). Data are expressed as the mean \pm S.E.M. of at least three independent experiments for B and D. ${ }^{* * *} P<0.001$; Scale bar: $20 \mu \mathrm{m}$. MII, metaphase II; ROS, reactive oxygen species.

Previous studies found that the complex cellular aging process was tightly linked to the action of sirtuins (Mair \& Dillin 2008, Alavez et al. 2011). In this study, we found that the Sirt 1, 2 and 3 and mRNA levels were significantly decreased in the aged oocyte, which was consistent with early studies (Zhang et al. 2014, 2016). Furthermore, only SIRT1 expression was significantly upregulated by melatonin treatment (Fig. 6A). Nevertheless, melatonin administration seemed to have little effect on the expression of Sirt2 and Sirt3 (Fig. 6B and C), which indicated that the SIRT1 may be an important target that mediates the protective effect of melatonin on delaying oocyte aging. Meanwhile, one recent study reported that long-term melatonin treatment can delay ovarian aging by multiple mechanisms, including the upregulation of SIRT1 (Kang et al. 2009). The SIRT1 inhibitor EX527 was used to confirm the function of the SIRT1 during oocyte aging, and the data showed that the ROS level and abnormal spindle morphology rate were significantly higher in oocytes receiving combined treatment with EX527 and melatonin than they were in oocytes treated with melatonin alone (Fig. 7), indicating that the protective effect of melatonin was markedly reduced. Moreover, when the SIRT1 activator SRT 1720 was used, we found that the oocyte aging was demonstrably delayed by the reduced ROS content and meiotic defects in oocytes than that of aged oocytes (Fig. 8).

Previous studies illustrated that melatonin is a direct free radical scavenger that protects the oocytes by avoiding damage from oxygen-free radicals (Tamura et al. 2008, Zhao et al. 2016). Some other studies found that melatonin also regulated the gene transcription of antioxidant enzymes (Pi et al. 2015, Han et al. 2017). An early study showed that the gene expression of SIRT1 was modulated by the oxidative stress (Prozorovski et al. 2008). The elevated ROS level will decrease the SIRT1 

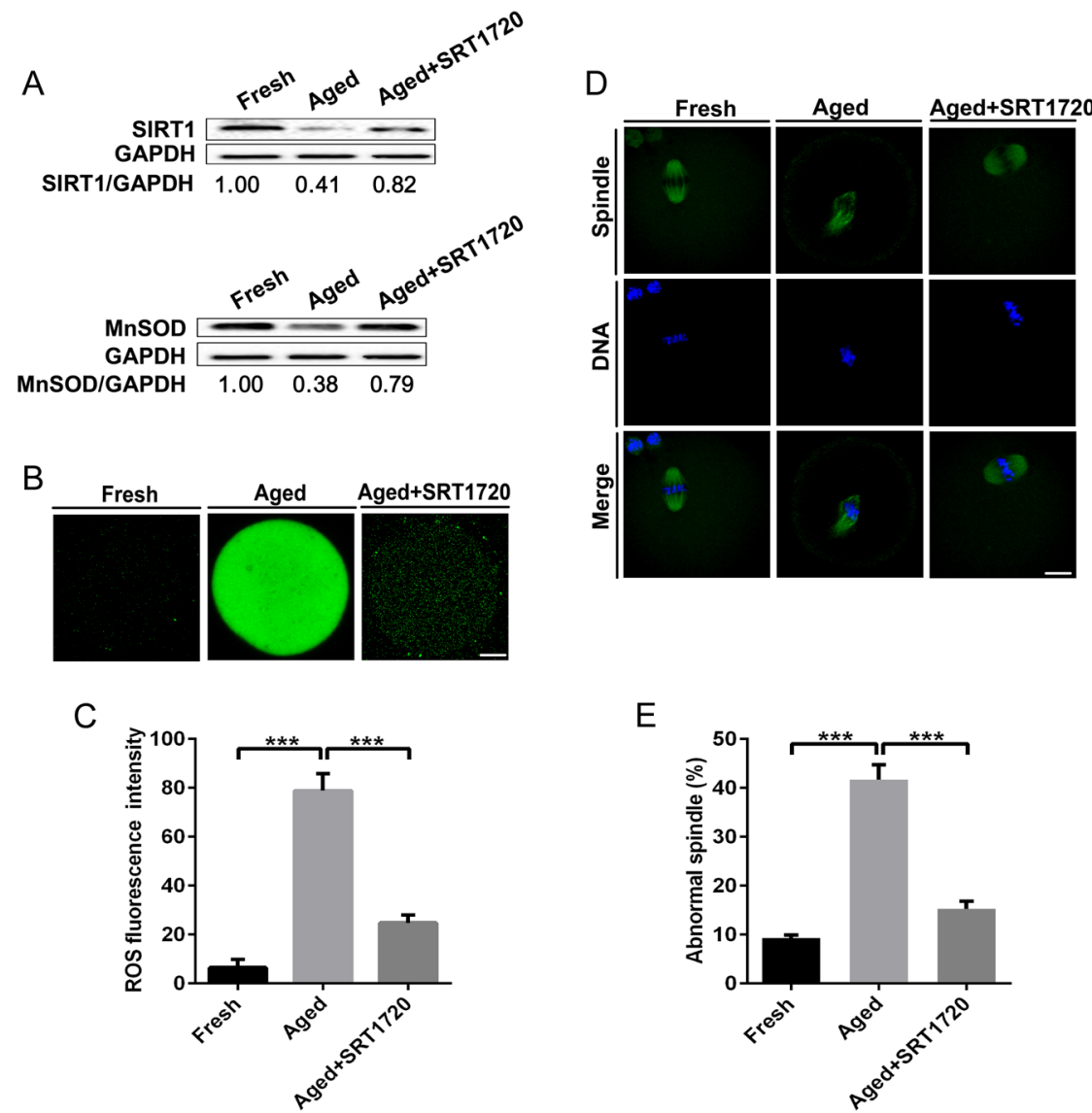

Figure 8 Activation of SIRT1 delays postovulatory aged oocyte aging by reducing the ROS level and abnormal spindle morphology. (A) The SIRT1 and MnSOD protein expression levels were analyzed by western blot in fresh, aged and SRT1720-treated aged oocytes. For each band, at least 300 oocytes were used. (B) Representative images of ROS fluorescence in MIl oocytes from the three different groups. (C) ROS fluorescence pixel intensity was evaluated in oocytes for each group ( $n=28$ for Fresh, $n=27$ Aged and $n=24$ for Aged+SRT1720). (D) The spindle morphology was assessed in each group, and the percentage of abnormal spindles was calculated for each group ( $n=85$ for Fresh, $n=92$ Aged and $n=79$ for Aged+SRT1720). Data are expressed as the mean \pm S.E.M. of at least three independent experiments for B and D. ${ }^{* * *} P<0.001$; Scale bar: $20 \mu \mathrm{m}$. MII, metaphase II; ROS, reactive oxygen species.

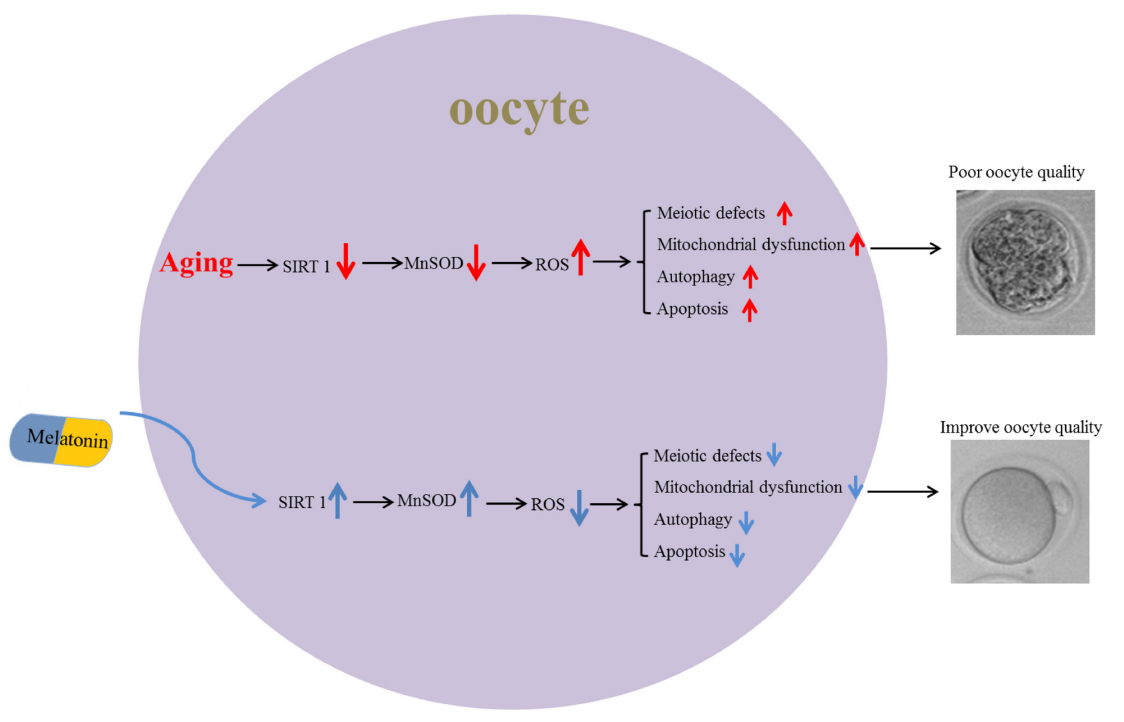

Figure 9 Working model of melatonin on delaying postovulatory oocyte aging. With the postovulatory oocyte aging, the expression of SIRT1 and MnSOD decrease, which leads to an increase in the ROS levels, thereby damaging the oocyte and resulting in elevated meiotic defects, mitochondrial dysfunction and the initiation of autophagy and apoptosis. However, melatonin supplementation could induce SIRT1 expression, which upregulates MnSOD protein expression in postovulatory aged oocytes, thus preventing oocyte aging, and improving the oocyte quality. ROS, reactive oxygen species. 
expression (Yang et al. 2007). All of the results indicated that the SIRT1 may be an important target for preventing oocyte aging. In this study, we found that the effect of melatonin on delaying postovulatory aging depended mainly on the upregulation of the SIRT1-mediated antioxidant enzyme MnSOD expression. Recently, accumulating evidence has suggested that melatonin could activate SIRT1 expression, which is related to the reduced ROS level, anti-autophagy and apoptotic effects in mammals (Yang et al. 2015, Zhao et al. 2015) and could then activate the expression of oxidative stress genes, including glutathione peroxidase, CAT and MnSOD (Zhong \& Mostoslavsky 2011, Emamgholipour et al. 2016). The results of these studies strongly suggest that melatonin has a stimulatory regulation of the SIRT1 expression to protect cells against the oxidative stress.

In summary, our research demonstrates that melatonin can significantly decrease the ROS levels and inhibit meiosis defects, the mitochondrial dysfunction, autophagy and apoptotic events by upregulating the SIRT1 and MnSOD protein levels in postovulatory aged oocytes (Fig. 9), indicating that melatonin supplementation is a promising treatment for preventing the postovulatory oocyte aging. However, additional studies are needed to evaluate the therapeutic utility of melatonin for delaying oocyte aging and improving the oocyte quality during the infertility treatment via ART in clinical practice.

\section{Declaration of interest}

The authors declare that there is no conflict of interest that could be perceived as prejudicing the impartiality of the research reported.

\section{Funding}

This study was supported by National Science Foundation for Young Scientists of China (Grants 31401274 and 81501332) and National Natural Science Foundation of China (Grant 31471404).

\section{References}

Alavez S, Vantipalli MC, Zucker DJ, Klang IM \& Lithgow GJ 2011 Amyloidbinding compounds maintain protein homeostasis during ageing and extend lifespan. Nature 472 226-229. (https://doi.org/10.1038/ nature09873)

Brzezinski A, Seibel MM, Lynch HJ, Deng MH \& Wurtman RJ 1987 Melatonin in human preovulatory follicular fluid. Journal of Clinical Endocrinology and Metabolism 64 865-867. (https://doi.org/10.1210/ jcem-64-4-865)

Coelho LA, Peres R, Amaral FG, Reiter RJ \& Cipolla-Neto J 2015 Daily differential expression of melatonin-related genes and clock genes in rat cumulus-oocyte complex: changes after pinealectomy. Journal of Pineal Research 58 490-499. (https://doi.org/10.1111/jpi.12234)

Dai X, Lu Y, Zhang M, Miao Y, Zhou C, Cui Z \& Xiong B 2017 Melatonin improves the fertilization ability of post-ovulatory aged mouse oocytes by stabilizing ovastacin and Juno to promote sperm binding and fusion. Human Reproduction 32 598-606. (https://doi.org/10.1093/humrep/ dew362)
Ducibella T, Duffy P, Reindollar R \& Su B 1990 Changes in the distribution of mouse oocyte cortical granules and ability to undergo the cortical reaction during gonadotropin-stimulated meiotic maturation and aging in vivo. Biology of Reproduction 43 870-876. (https://doi.org/10.1095/ biolreprod43.5.870)

Emamgholipour S, Hossein-Nezhad A, Sahraian MA, Askarisadr F \& Ansari M 2016 Evidence for possible role of melatonin in reducing oxidative stress in multiple sclerosis through its effect on SIRT1 and antioxidant enzymes. Life Sciences 145 34-41. (https://doi.org/10.1016/j.lfs.2015.12.014)

Galano A, Tan DX \& Reiter RJ 2011 Melatonin as a natural ally against oxidative stress: a physicochemical examination. Journal of Pineal Research 51 1-16. (https://doi.org/10.1111/j.1600-079X.2011.00916.x)

Giritharan G, Talbi S, Donjacour A, Di Sebastiano F, Dobson AT \& Rinaudo PF 2007 Effect of in vitro fertilization on gene expression and development of mouse preimplantation embryos. Reproduction 134 63-72. (https://doi.org/10.1530/REP-06-0247)

Guo JY, Li F, Wen YB, Cui HX, Guo ML, Zhang L, Zhang YF, Guo YJ \& Guo YX 2017 Melatonin inhibits Sirt1-dependent NAMPT and NFAT5 signaling in chondrocytes to attenuate osteoarthritis. Oncotarget 8 55967-55983. (https://doi.org/10.18632/oncotarget.23705)

Han D, Huang W, Li X, Gao L, Su T, Li X, Ma S, Liu T, Li C, Chen J et al. 2016 Melatonin facilitates adipose-derived mesenchymal stem cells to repair the murine infarcted heart via the SIRT1 signaling pathway. Journal of Pineal Research 60 178-192. (https://doi.org/10.1111/jpi.12299)

Han L, Wang H, Li L, Li X, Ge J, Reiter RJ \& Wang Q 2017 Melatonin protects against maternal obesity-associated oxidative stress and meiotic defects in oocytes via the SIRT3-SOD2-dependent pathway. Journal of Pineal Research 63. (https://doi.org/10.1111/jpi.12431)

Ishizuka B, Kuribayashi Y, Murai K, Amemiya A \& Itoh MT 2000 The effect of melatonin on in vitro fertilization and embryo development in mice. Journal of Pineal Research 28 48-51. (https://doi.org/10.1034/j.1600079x.2000.280107.x)

Kang JT, Koo OJ, Kwon DK, Park HJ, Jang G, Kang SK \& Lee BC 2009 Effects of melatonin on in vitro maturation of porcine oocyte and expression of melatonin receptor RNA in cumulus and granulosa cells. Journal of Pineal Research 46 22-28. (https://doi.org/10.1111/j.1600-079X.2008.00602.x)

Kops GJ, Dansen TB, Polderman PE, Saarloos I, Wirtz KW, Coffer PJ, Huang TT, Bos JL, Medema RH \& Burgering BM 2002 Forkhead transcription factor $\mathrm{FOXO3a}$ protects quiescent cells from oxidative stress. Nature 419 316-321. (https://doi.org/10.1038/nature01036)

Kosubek A, Klein-Hitpass L, Rademacher K, Horsthemke B \& Ryffel GU 2010 Aging of Xenopus tropicalis eggs leads to deadenylation of a specific set of maternal mRNAs and loss of developmental potential. PLOS ONE 5 e13532. (https://doi.org/10.1371/journal.pone.0013532)

Liu M, Yin Y, Ye X, Zeng M, Zhao Q, Keefe DL \& Liu L 2013 Resveratrol protects against age-associated infertility in mice. Human Reproduction 28 707-717. (https://doi.org/10.1093/humrep/des437)

Liu Z, Gan L, Zhang T, Ren Q \& Sun C 2017 Melatonin alleviates adipose inflammation through elevating alpha-ketoglutarate and diverting adipose-derived exosomes to macrophages in mice. Journal of Pineal Research 64. (https://doi.org/10.1111/jpi.12455)

Lord T \& Aitken RJ 2013 Oxidative stress and ageing of the post-ovulatory oocyte. Reproduction 146 R217-R227. (https://doi.org/10.1530/REP-130111)

Lord T, Nixon B, Jones KT \& Aitken RJ 2013 Melatonin prevents postovulatory oocyte aging in the mouse and extends the window for optimal fertilization in vitro. Biology of Reproduction 88 67. (https://doi. org/10.1095/biolreprod.112.106450)

Mair W \& Dillin A 2008 Aging and survival: the genetics of life span extension by dietary restriction. Annual Review of Biochemistry 77 727-754. (https://doi.org/10.1146/annurev.biochem.77.061206.171059)

Miao YL, Kikuchi K, Sun QY \& Schatten H 2009 Oocyte aging: cellular and molecular changes, developmental potential and reversal possibility. Human Reproduction Update 15 573-585. (https://doi.org/10.1093/ humupd/dmp014)

Nopparat C, Sinjanakhom P \& Govitrapong P 2017 Melatonin reverses H2 $\mathrm{O} 2$-induced senescence in $\mathrm{SH}-\mathrm{SY} 5 \mathrm{Y}$ cells by enhancing autophagy via sirtuin 1 deacetylation of the RelA/p65 subunit of NF-kappaB. Journal of Pineal Research 63. (https://doi.org/10.1111/jpi.12407)

Papis K, Poleszczuk O, Wenta-Muchalska E \& Modlinski JA 2007 Melatonin effect on bovine embryo development in vitro in relation to oxygen concentration. Journal of Pineal Research 43 321-326. (https:// doi.org/10.1111/j.1600-079X.2007.00479.x) 
Pi H, Xu S, Reiter RJ, Guo P, Zhang L, Li Y, Li M, Cao Z, Tian L, Xie J et al. 2015 SIRT3-SOD2-mROS-dependent autophagy in cadmium-induced hepatotoxicity and salvage by melatonin. Autophagy 11 1037-1051. (https://doi.org/10.1080/15548627.2015.1052208)

Prozorovski T, Schulze-Topphoff U, Glumm R, Baumgart J, Schroter $F_{,}$ Ninnemann O, Siegert E, Bendix I, Brustle O, Nitsch R et al. 2008 Sirt1 contributes critically to the redox-dependent fate of neural progenitors. Nature Cell Biology 10 385-394. (https://doi.org/10.1038/ncb1700)

Reiter RJ 1980 The pineal and its hormones in the control of reproduction in mammals. Endocrine Reviews 1 109-131. (https://doi.org/10.1210/ edrv-1-2-109)

Reiter RJ 1991 Pineal melatonin: cell biology of its synthesis and of its physiological interactions. Endocrine Reviews 12 151-180. (https://doi. org/10.1210/edrv-12-2-151)

Reiter RJ 1998 Melatonin and human reproduction. Annals of Medicine 30 103-108. (https://doi.org/10.3109/07853899808999391)

Rodriguez-Osorio N, Kim IJ, Wang H, Kaya A \& Memili E 2007 Melatonin increases cleavage rate of porcine preimplantation embryos in vitro. Journal of Pineal Research 43 283-288. (https://doi.org/10.1111/j.1600079X.2007.00475.x)

Ronnberg L, Kauppila A, Leppaluoto J, Martikainen H \& Vakkuri O 1990 Circadian and seasonal variation in human preovulatory follicular fluid melatonin concentration. Journal of Clinical Endocrinology and Metabolism 71 492-496.

Roy D \& Belsham DD 2002 Melatonin receptor activation regulates GnRH gene expression and secretion in GT1-7 GnRH neurons. Signal transduction mechanisms. Journal of Biological Chemistry 277 251-258. (https://doi.org/10.1074/jbc.M108890200)

Shi JM, Tian XZ, Zhou GB, Wang L, Gao C, Zhu SE, Zeng SM, Tian JH \& Liu GS 2009 Melatonin exists in porcine follicular fluid and improves in vitro maturation and parthenogenetic development of porcine oocytes. Journal of Pineal Research 47 318-323. (https://doi.org/10.1111/j.1600079X.2009.00717.x)

Song C, Zhao J, Fu B, Li D, Mao T, Peng W, Wu H \& Zhang Y 2017 Melatonin-mediated upregulation of Sirt3 attenuates sodium fluorideinduced hepatotoxicity by activating the MT1-PI3K/AKT-PGC-1alpha signaling pathway. Free Radical Biology and Medicine 112 616-630. (https://doi.org/10.1016/j.freeradbiomed.2017.09.005)

Sun QY, Miao YL \& Schatten H 2009 Towards a new understanding on the regulation of mammalian oocyte meiosis resumption. Cell Cycle $\mathbf{8}$ 2741-2747. (https://doi.org/10.4161/cc.8.17.9471)

Takahashi T, Takahashi E, Igarashi H, Tezuka N \& Kurachi H 2003 Impact of oxidative stress in aged mouse oocytes on calcium oscillations at fertilization. Molecular Reproduction and Development 66 143-152. (https://doi.org/10.1002/mrd.10341)

Tamura H, Takasaki A, Miwa I, Taniguchi K, Maekawa R, Asada H, Taketani T, Matsuoka A, Yamagata Y, Shimamura K et al. 2008 Oxidative stress impairs oocyte quality and melatonin protects oocytes from free radical damage and improves fertilization rate. Journal of Pineal Research 44 280-287. (https://doi.org/10.1111/j.1600-079X.2007.00524.x)

Tamura H, Kawamoto M, Sato S, Tamura I, Maekawa R, Taketani T, Aasada H, Takaki E, Nakai A, Reiter RJ et al. 2017 Long-term melatonin treatment delays ovarian aging. Journal of Pineal Research 62. (https:// doi.org/10.1111/jpi.12381)

Tarin JJ, Perez-Albala S, Aguilar A, Minarro J, Hermenegildo C \& Cano A 1999 Long-term effects of postovulatory aging of mouse oocytes on offspring: a two-generational study. Biology of Reproduction $\mathbf{6 1}$ 1347-1355. (https://doi.org/10.1095/biolreprod61.5.1347)

Tatone C, Di Emidio G, Vitti M, Di Carlo M, Santini S Jr, D'Alessandro AM, Falone S \& Amicarelli F 2015 Sirtuin functions in female fertility: possible role in oxidative stress and aging. Oxidative Medicine and Cellular Longevity 2015 659687. (https://doi.org/10.1155/2015/659687)

Tomas-Zapico C \& Coto-Montes A 2005 A proposed mechanism to explain the stimulatory effect of melatonin on antioxidative enzymes. Journal of Pineal Research 39 99-104. (https://doi.org/10.1111/j.1600079X.2005.00248.x)

Tong J, Sheng S, Sun Y, Li H, Li WP, Zhang C \& Chen ZJ 2017 Melatonin levels in follicular fluid as markers for IVF outcomes and predicting ovarian reserve. Reproduction 153 443-451. (https://doi.org/10.1530/ REP-16-0641)
Wakayama S, Thuan NV, Kishigami S, Ohta H, Mizutani E, Hikichi T, Miyake M \& Wakayama T 2004 Production of offspring from one-dayold oocytes stored at room temperature. Journal of Reproduction and Development 50 627-637. (https://doi.org/10.1262/jrd.50.627)

Wang Q \& Sun QY 2007 Evaluation of oocyte quality: morphological, cellular and molecular predictors. Reproduction, Fertility, and Development 19 1-12. (https://doi.org/10.1071/RD06103)

Wang F, Tian X, Zhang L, Tan D, Reiter RJ \& Liu G 2013 Melatonin promotes the in vitro development of pronuclear embryos and increases the efficiency of blastocyst implantation in murine. Journal of Pineal Research 55 267-274. (https://doi.org/10.1111/jpi.12069)

Wang F, Tian X, Zhang L, Gao C, He C, Fu Y, Ji P, Li Y, Li N \& Liu G 2014 Beneficial effects of melatonin on in vitro bovine embryonic development are mediated by melatonin receptor 1. Journal of Pineal Research 56 333-342. (https://doi.org/10.1111/jpi.12126)

Wang H, Jo YJ, Oh JS \& Kim NH 2017a Quercetin delays postovulatory aging of mouse oocytes by regulating SIRT expression and MPF activity. Oncotarget 8 38631-38641. (https://doi.org/10.18632/ oncotarget.16219)

Wang T, Gao YY, Chen L, Nie ZW, Cheng W, Liu X, Schatten H, Zhang X \& Miao YL 2017b Melatonin prevents postovulatory oocyte aging and promotes subsequent embryonic development in the pig. Aging $\mathbf{9}$ 1552-1564. (https://doi.org/10.18632/ aging.101252)

Xu Z, Abbott A, Kopf GS, Schultz RM \& Ducibella T 1997 Spontaneous activation of ovulated mouse eggs: time-dependent effects on M-phase exit, cortical granule exocytosis, maternal messenger ribonucleic acid recruitment, and inositol 1,4,5-trisphosphate sensitivity. Biology of Reproduction 57 743-750. (https://doi.org/10.1095/biolreprod57.4.743)

Yang Y, Fu W, Chen J, Olashaw N, Zhang X, Nicosia SV, Bhalla K \& Bai W 2007 SIRT1 sumoylation regulates its deacetylase activity and cellular response to genotoxic stress. Nature Cell Biology 9 1253-1262. (https:// doi.org/10.1038/ncb1645)

Yang Y, Jiang S, Dong Y, Fan C, Zhao L, Yang X, Li J, Di S, Yue L, Liang G et al. 2015 Melatonin prevents cell death and mitochondrial dysfunction via a SIRT1-dependent mechanism during ischemic-stroke in mice. Journal of Pineal Research 58 61-70. (https://doi.org/10.1111/jpi.12193)

Zhang L, Hou X, Ma R, Moley K, Schedl T \& Wang Q 2014 Sirt2 functions in spindle organization and chromosome alignment in mouse oocyte meiosis. FASEB Journal 28 1435-1445. (https://doi.org/10.1096/fj.13244111)

Zhang T, Zhou Y, Li L, Wang HH, Ma XS, Qian WP, Shen W, Schatten H \& Sun QY 2016 SIRT1, 2, 3 protect mouse oocytes from postovulatory aging. Aging 8 685-696. (https://doi.org/10.18632/aging.100911)

Zhai M, Li B, Duan W, Jing L, Zhang B, Zhang M, Yu L, Liu Z, Yu B, Ren K et al. 2017 Melatonin ameliorates myocardial ischemia reperfusion injury through SIRT3-dependent regulation of oxidative stress and apoptosis. Journal of Pineal Research 63. (https://doi.org/10.1111/ jpi.12419)

Zhao L, An R, Yang Y, Yang X, Liu H, Yue L, Li X, Lin Y, Reiter RJ \& Qu Y 2015 Melatonin alleviates brain injury in mice subjected to cecal ligation and puncture via attenuating inflammation, apoptosis, and oxidative stress: the role of SIRT1 signaling. Journal of Pineal Research 59 230-239. (https://doi.org/10.1111/jpi.12254)

Zhao XM, Hao HS, Du WH, Zhao SJ, Wang HY, Wang N, Wang D, Liu Y, Qin T \& Zhu HB 2016 Melatonin inhibits apoptosis and improves the developmental potential of vitrified bovine oocytes. Journal of Pineal Research 60 132-141. (https://doi.org/10.1111/jpi.12290)

Zhong L \& Mostoslavsky R 2011 Fine tuning our cellular factories: sirtuins in mitochondrial biology. Cell Metabolism 13 621-626. (https://doi. org/10.1016/j.cmet.2011.05.004)

Received 23 April 2018

First decision 4 May 2018

Revised manuscript received 9 May 2018

Accepted 11 May 2018 